\title{
Slimmed down for sensitivity
}

IEEE Electron Device Lett. https://doi.org/10.1109/

LED.2018.2846184 (2018)

Wearable sensors that can detect vital signs could be used to continuously monitor a person's health. The devices should be flexible and offer high sensitivity. They also typically require a constant power supply, which can restrict their broader application. Lead zirconate titanate (PZT) - a perovskite ceramic that is able to translate mechanical strain into electrical signals - is a promising material for self-powered applications. However, its low sensitivity has limited its use in wearable sensors. Bin Yang and colleagues at Shanghai Jiao Tong University have now developed a selfpowered PZT force sensor that can detect artery pulse signals in real time and recognize hand gestures.

The researchers first bonded a PZT layer to a beryllium copper foil in order to help create a uniform strain distribution in the device. Next, the PZT layer was thinned down to a thickness of $50 \mu \mathrm{m}$ to reduce the mechanical stress on the metal layer. Finally, the PZT layer was bonded to a flexible plastic substrate. The resulting sensor exhibited a high sensitivity of up to $10 \mathrm{~V} \mathrm{~N}^{-1}$ and could be attached to curved surfaces. To further assess its performance, the sensor was placed on a person's wrist, arm and neck, and pulse measurements were recorded. The sensor can also be connected to a computer via a wireless signal transmission system.

Christiana Varnava

Published online: 13 July 2018

https://doi.org/10.1038/s41928-018-0110-4 